\title{
Stimulus discriminability, contingency discriminability, and schedule performance
}

\author{
MICHAEL DAVISON \\ University of Auckland, Auckland, New Zealand \\ and \\ PETER E. JENKINS \\ Vassar College, Poughkeepsie, New York
}

\begin{abstract}
A detection-theory model to describe the effects of varying stimulus disparity on switching-key concurrent variable-interval schedule performance (Miller, Saunders, \& Bourland, 1980) is presented. It describes the available data on stimulus disparity well. Using the additional notion of contingency discriminability, the model is then developed into an account of schedule and stimulus control that is both wider in application and conceptually clearer than the generalized matching law. A basic assumption of the new model is that subjects may not perfectly discriminate that a reinforcer followed a response of one class versus that of another, and this ability is measured as reinforcer-contingency discriminability, $d_{r}$. This idea is then applied to performance in signal-detection procedures, both with and without error reinforcement, to multiple-schedule performance, and to single-schedule performance. The model fitted the data well, and it thus constitutes a coherent and viable alternative to the generalized matching law in the procedures and conditions in which the latter has been shown to apply.
\end{abstract}

\section{Stimulus Disparity in Concurrent Schedules}

The distribution of responses between the alternatives in concurrent variable-interval (VI) schedules is very well described by the generalized matching law (Baum, 1974). This is written:

$$
\frac{B_{1}}{B_{2}}=c\left(\frac{R_{1}}{R_{2}}\right)^{a}
$$

where $B$ is the behavior emitted and $R$ is the reinforcers obtained on the two schedules subscripted 1 and 2 . The parameter $\mathbf{c}$ is called bias, and it measures a constant proportional preference for one alternative due to a constant differential between some variables that affect choice but are constant and unequal during the experiment. The parameter a is called "sensitivity to reinforcement" (Lobb \& Davison, 1975), and it measures the rate of change in the behavior ratio with changes in the reinforcer ratio. The value of a is usually about .8 (Baum, 1979).

Miller, Saunders, and Bourland (1980) showed that the

Most of the conceptual work on the model presented here was done while Peter E. Jenkins was a postdoctoral fellow at Auckland University. We thank Dianne McCarthy for stimulating discussions, Tony Nevin and John Wearden for their valuable comments on versions of this work, and Peter Killeen for the random-leap multidimensional nonlinear curvefitting program used extensively here. We also thank the New Zealand University Grants Committee, both for their support of Peter E. Jenkins and for equipment grants to the first author. Reprints may be obtained from Michael Davison, Psychology Department, Auckland University, Private Bag, Auckland, New Zealand, or from Peter E. Jenkins, Department of Psychology, Vassar College, Poughkeepsie, New York 12601. value of $\mathbf{a}$ in Equation 1 was a function of the difference between the stimuli signaling the two concurrent-schedule components. Using a switching-key procedure (Findley, 1958), the components were signaled by two different line orientations displayed on the pecking key. The difference between the orientations was $0^{\circ}$ for two subjects, $15^{\circ}$ for two subjects, and $45^{\circ}$ for two subjects. For each stimulus separation, the component VI schedules were varied to give six or seven different reinforcer ratios. Miller et al. reported a values of .17 and .17 for the $0^{\circ}$ separation, .28 and .37 for the $15^{\circ}$ separation, and .97 and 1.00 for the $45^{\circ}$ separation.

It is evident from Equation 1 that if a subject could not discriminate a response on one schedule from a response on the other, the value of a must be 0 . This situation provides the lower limit for the expected value of a, although Rachlin, Kagel, and Battalio (1980) have argued that negative a values are logically possible in some circumstances. The upper limit of a is not logically predictable, and would appear to be infinite. However, empirical research (Baum, 1979; Wearden \& Burgess, 1982) has shown that a values greater than 1 seldom occur in the conventional concurrent-schedule procedure. When, however, changing over between the schedules is constrained, such that the changeover performance itself is not measured as part of the concurrent-schedule performance, overmatching (a $>1)$ can occur reliably. Examples of this are: when a fixedratio changeover is required, and the fixed-ratio performance is not counted as part of the concurrent-schedule response allocation (e.g., Pliskoff \& Fetterman, 1981; see also Taylor \& Davison, 1983, for an analysis that includes 
the ratio performance); when travel is required for a changeover (Baum, 1982); and when changeovers are punished (e.g., Todorov, 1971). The present model does not, as yet, attempt to handle such constraints. However, if the contingencies involved in changing over are punishing (either by shock or by a period of time out from reinforcers), the models of punishment offered by de Villiers (1980) and Farley (1980) might be used in conjunction with the present model to predict overmatching. Dealing with this possibility here would needlessly complicate the initial model presentation.

We may conceptualize that, in a concurrent schedule, the subject's task is to decide, after each reinforcer delivery, which of the two response classes produced that reinforcer. Thus, in detection-theory terms, the stimuli to be detected are the delivery of reinforcers for one response $\left(R_{1}\right)$ and the delivery of those for the other response $\left(R_{2}\right)$. We propose that the subject allocates these reinforcers, according to its ability and to the current environmental conditions, to the two response classes $B_{1}$ and $B_{2}$. If these are easily discriminable classes (e.g., emitted in the presence of clearly differentiable environmental events), the subject will allocate the reinforcers to the response classes accurately. If, however, they are poorly discriminable classes, the subject will be inaccurate, with the limiting condition of no discriminability resulting in equally frequent allocation of reinforcers to the two experimenter-defined classes. The subject will thus show a varying discrimination of the contingencies of reinforcement according to the discriminability of the signaling conditions. If we accept, as discussed above, that $0<\mathrm{a}<1$, a model that describes the effects of the discriminability of the signaling stimuli on conventional concurrentschedule performance is:

$$
\frac{B_{1}}{B_{2}}=c\left(\frac{d_{1} R_{1}+R_{2}}{d_{r} R_{2}+R_{1}}\right)
$$

Here, the number of parameters is the same as in Equation 1 , but $a$ is replaced by a parameter $d_{r}$ which measures the discriminability of the operant classes signaled by the two stimuli. $d_{r}$, which is dimensionless, will vary between 1 (no contingency discriminability) and $\infty$ (perfect contingency discriminability). This measure is closely related to the discriminability measure defined in the Davison and Tustin (1978) signal-detection model (log d, the logarithm of the above measure), and to $\mathrm{d}^{\prime}$ in signaldetection theory (e.g., Green \& Swets, 1974). The scale transform of $d_{r}$ to $d^{\prime}$ is just the $z$-transform of $d_{r} /\left(1+d_{r}\right)$.

It might be objected immediately that Equation 2 cannot be correct as the reinforcers in that equation sum to $\left(R_{1}+R_{2}\right)\left(1+d_{1}\right)$, more than the total number of reinforcers delivered $\left(\mathbf{R}_{1}+\mathbf{R}_{\mathbf{2}}\right)$. A conceptually complete version of Equation 2 is:

$$
\frac{B_{1}}{B_{2}}=\frac{\left.\left.\frac{d_{r} R_{1}}{\left(1+d_{r}\right.}\right)+\frac{R_{2}}{\left(1+d_{r}\right.}\right)}{\left.\left(\frac{d_{r} R_{2}}{1+d_{r}}\right)+\frac{R_{1}}{\left(1+d_{r}\right.}\right)}
$$

This equation immediately reduces to Equation 2, but the reinforcer total is $R_{1}+R_{2}$.

In the above development, we have subscripted the discriminability measure, $d_{r}$, for good reason. In a switchingkey concurrent schedule, $d_{r}$ measures the discriminability of the currently available alternatives, which are signaled by the discriminative stimuli on the main key. If these two stimuli are identical, $d_{r}$ will be 1 . Equally, on a two-key concurrent schedule, $d_{r}$ measures the discriminability of the alternatives, which are "signaled" by left and right keys. The degree to which a subject misallocates a delivered reinforcer (due to a failure in the discriminability between the response-reinforcer contingencies) to the alternate schedule or key is measured by $d_{r}$. Later, we shall use $d_{s}$ to denote stimulus discriminability in procedures in which discriminative stimuli are employed in successive-scheduling procedures.

Empirically, Equation 2 deals nicely with Miller et al.'s data. The least square estimates of $d_{r}$ and $c$ are given in Table 1. Notice, however, that $d_{r}$ was not zero for the $0^{\circ}$ separation data (a in Equation 1 was not zero either). This was, most likely, due to the subjects' discriminating the components to some extent on the basis of the obtained reinforcer rates on some kind of win-stay looseshift strategy. Also shown in Table 1 are estimates of a and $\mathbf{c}$ in Equation 1 obtained by using linear regression with the data given by Miller et al. We are unsure of why

Table 1

Least Squares Estimates of the Parameters of Equations 1 and 2 Using the Data Reported by Miller, Saunders, and Bourland (1980)

\begin{tabular}{lcccc}
\hline & & Equation 1 & & \\
Bird & $\begin{array}{c}\text { Separation } \\
\text { (degrees) }\end{array}$ & $\mathbf{a}$ & $\mathbf{c}$ & VAC \\
\hline 523 & 0 & $.16(.17)$ & 1.08 & .72 \\
518 & 0 & $.18(.17)$ & 1.02 & .62 \\
515 & 15 & $.42(.37)$ & 1.50 & .65 \\
516 & 15 & $.26(.28)$ & .97 & .72 \\
517 & 45 & $.99(1.0)$ & 1.04 & .89 \\
525 & 45 & $.83(.97)$ & .89 & .82 \\
\hline
\end{tabular}

\begin{tabular}{lcccc}
\hline Bird & $\begin{array}{c}\text { Separation } \\
\text { (degrees) }\end{array}$ & $\mathrm{d}_{\mathrm{r}}$ & $\mathrm{c}$ & VAC \\
\hline 523 & 0 & 1.49 & 1.09 & .74 \\
518 & 0 & 1.53 & 1.02 & .59 \\
515 & 15 & 3.06 & 1.51 & .72 \\
516 & 15 & 1.83 & .97 & .72 \\
517 & 45 & 57.78 & 1.06 & .90 \\
525 & 45 & 19.54 & .84 & .85 \\
\hline
\end{tabular}

Note-VAC is the proportion of data variance accounted for. Estimates of $\mathbf{a}$, reported by Miller et al., are shown in parentheses. 
these parameter estimates are somewhat different from those reported by Miller et al., which are also shown in Table 1. Table 1 shows that Equation 2 fits the Miller et al. data on stimulus disparity marginally better than does Equation 1.

Equation 2 may deal with more than just the direct effects of stimulus separation on concurrent-schedule performances. It has the mathematical property of mimicking, over the usual range of reinforcer ratios, the generalized matching law power function (Equation 1). This is displayed in Table 2, in which for a set of reinforcer ratios ranging from .1 to 10, Equation 2 was fit to data produced by particular values of $\mathbf{a}$, ranging from .1 to .9. None of the fits of Equation 2 to Equation 1 gave proportions of variance accounted for of less than .99 . Thus, Equation 2 will fit concurrent VI VI schedule data as well as does Equation 1 over the usual range of reinforcer ratios. Algebraically, therefore, Equation 2 is a strong competitor for Equation 1. Beyond the usually investigated range of obtained reinforcer ratios (about .1 to 10$)$, Equation 2 predicts lower preference than does the generalized matching law (Equation 1). No data are available to determine which equation fits best in these extreme regions.

The parameter $a$ in Equation 1 (sensitivity to reinforcement) has been shown to have formal justification (Allen, 1981; Prelec, 1984). However, the parameter $d_{r}$ in Equation 2 (which we will term contingency discriminability) has a clearer conceptual interpretation than does the parameter a (sensitivity to reinforcement) in Equation 1. Equation 1 asserts that the obtained reinforcer ratio is scaled on input by the subject, or possibly that the veridical input is scaled on output by the subject. There is no generally accepted reason for this scaling, although Wearden (1980) related it to the psychophysical scaling of time. Equation 2, on the other hand, provides a reason: The subject may fail (because of poor stimulus control) to allocate the obtained reinforcers accurately to the emitted response class (by which we mean, of course, the discriminatively controlled response). Thus, if the subject was asked (using a detection procedure) after a reinforcer delivery, "From which discriminative stimulus was that reinforcer obtained?", it should show a degree of inaccuracy, dependent on the level of stimulus discrimina-

Table 2

Fits of Equation 2 to Data Produced Using Equation 1 Over a Range of Reinforcer Ratios from .1 to 10 , and Using Various a Values

\begin{tabular}{ccc}
\hline a Value & Obtained d & VAC by Equation 2 \\
\hline .1 & 1.29 & .99 \\
.3 & 2.17 & .99 \\
.5 & 3.93 & .99 \\
.6 & 5.59 & .99 \\
.7 & 8.53 & 1.00 \\
.8 & 14.91 & 1.00 \\
.9 & 37.69 & 1.00 \\
\hline
\end{tabular}

Note - A total of 50 reinforcers per hour and 2,000 responses per hour were assumed. $V A C$ is the proportion of the variance accounted for. bility. Equation 2 has the edge over Equation 1 in conceptual clarity.

A further empirical consideration shows Equation 2 to be superior to Equation 1. Equation 2 suggests that the maximum value of a will be 1.0 , but that any failure of discrimination of the alternatives and their reinforcer rates will decrease the value of a. Because asymptotic discriminability will seldom be achieved, a estimates should generally fall below 1.0 , but since researchers generally use highly discriminable stimuli, a should be close to 1.0. Statistical error in estimating a will produce a distribution of values around true values, which will occasionally result in a values greater than 1.0. Such a distribution, predicted from Equation 2, is exactly the distribution of empirical a values described by Baum (1979) and by Wearden and Burgess (1982). On this basis, Equation 2 is to be preferred over Equation 1 .

Equation 2 predicts that any independent variable that changes the discriminability of the components will affect $d_{r}$, and hence a in Equation 1. Miller et al. (1980) have described the direct effects of stimulus discriminability. Baum (1974) discussed similar effects, and also suggested that the differentiation of the components would be enhanced by longer changeover delays (periods after changing between schedules in which reinforcers are unavailable) (see also Baum, 1979, and de Villiers, 1977). Equation 2 makes these effects of changeover delay understandable. Trace, rather than continuous, stimulus presentations should decrease $d_{r}$ and $a$, and they do, at least in multiple schedules (White \& Redman, 1983). Different types of concurrently arranged schedules may be more or less discriminable. For example, concurrent fixedinterval VI schedules show a low a value than do concurrent VI VI schedules (Lobb \& Davison, 1975), and concurrent arithmetic VI schedules give lower a values than do concurrent exponential schedules (Taylor \& Davison, 1983). Even the delivery of reinforcers themselves has been shown to degrade stimulus discriminability (Williams, 1975). Indeed, any variables that have been shown to decrease discriminability in psychophysical and detection-theory paradigms would be expected to have a similar effect on concurrent-schedule performance and to decrease matching sensitivity.

Finally, when concurrent VI extinction schedules are arranged, subjects seldom emit no responses on the extinction schedule (e.g., Davison \& Hunter, 1976)-but Equation 1 must predict infinite response-rate ratios whatever the value of $\mathbf{a}$. If $\mathbf{R}_{\mathbf{2}}$ was 0 in Equation 2, however, that equation reduces to:

$$
\frac{B_{1}}{B_{2}}=\mathrm{cd}_{r}
$$

The ratio is noninfinite, as generally found except when either the discriminability $\left(d_{r}\right)$ or the bias (c) is infinite. Furthermore, Equation 3 predicts that the response ratio in stable concurrent reinforcement extinction performance 
will be constant when discriminability and bias are constant, and independent of the reinforcer rate. Nevin (1967) reported data that show that this prediction is correct in a discrete-trials concurrent discrimination procedure.

Some research directly concerned with the discrimination of response-reinforcer contingencies has been reported. Killeen $(1978,1981)$ used a signal-detection procedure to measure pigeons' abilities to report whether their own behavior produced a reinforcer, or whether the reinforcer was delivered independently of their behavior. Killeen (1978) reported data from which a $d_{r}$ value of about 6 might be estimated using nonlinear regression. Lattal (1975) also used a signal-detection procedure to investigate the ability of pigeons to discriminate two contingencies of reinforcement arranged during a sample component. The contingencies were a differentialreinforcement-of-low-rates schedule, in which responses were reinforced only if they were emitted more than $10 \mathrm{sec}$ after the previous response, versus a schedule in which reinforcers were delivered after $10 \mathrm{sec}$ if no response was emitted. The mean $d_{r}$ was 12 for Bird 62 and 6 for Bird 54.

The present approach to concurrent-schedule performance can be compared with that taken by Wearden (1983), who suggested that undermatching might derive from a certain finite probability of a subject's responding randomly, that is, without regard to the reinforcer contingencies. In Wearden's model, $\mathbf{p}$ is the probability that a burst of responses will be allocated according to strict (simple) matching ( $a=1$ in Equation 1), and the value of $\mathbf{p}$ must be some function of contingency discriminability. The nature of this function is not defined. Wearden's Equation 8 may be written:

$$
\frac{B_{1}}{B_{2}}=c\left[\frac{2 p R_{1}+(1-p)\left(R_{1}+R_{2}\right)}{2 p R_{2}+(1-p)\left(R_{1}+R_{2}\right)}\right] .
$$

Equation $2 b$, here, may be written in probability terms as:

$$
\frac{B_{1}}{B_{2}}=c\left[\frac{p^{\prime} R_{1}+\left(1-p^{\prime}\right) R_{2}}{p^{\prime} R_{2}+\left(1-p^{\prime}\right) R_{1}}\right],
$$

where $\mathrm{p}^{\prime}$ is the probability of allocating a reinforcer to the correct response class, and $\left(1-p^{\prime}\right)$ is the probability of allocating a reinforcer to the alternative response class. The two equations are algebraically rather similar. The major differences between the two approaches may be summed up thus: Wearden's theory assumes that the subject emits some response bursts under the precise control of a strict matching law, and some bursts that are not controlled at all by the reinforcer contingencies. The present theory assumes that subjects always strictly match their response outputs to the (somewhat mutually confused) reinforcer frequencies. Thus, Wearden's model assumes an output failure reminiscent of behavioral oscillation, whereas the present model assumes a discrimination or input failure. One reason for preferring the present model is that, as we shall show, it leads to a coherent theory of both contingency and stimulus detectability in a range of different procedures.

\section{Signal Detection}

The behavioral detection model proposed by Davison and Tustin (1978) used the generalized matching law as the basic process which translates reinforcer-frequency differentials between outcomes into bias in behavior. Since we are here proposing to replace the generalized matching law with a contingency-detection process, we must investigate the effects of such a change on the DavisonTustin detection model.

The yes-no detection procedure is diagramed in Figure 1. In its simplest form, it consists of a multipleschedule arrangement in which, in the presence of the first stimulus, $B_{1}$ is reinforced and $B_{2}$ is under extinction and, in the presence of the second stimulus, $B_{2}$ is reinforced and $B_{1}$ is under extinction. Our conventional labeling of the four outcome cells is shown in Figure 1. Using the generalized matching law, and subscripting the variables as defined in Figure 1, Davison and Tustin wrote two equations. In $S_{1}$ :

$$
\frac{B_{w}}{B_{\mathbf{x}}}=\operatorname{dc}\left(\frac{R_{w}}{R_{z}}\right)^{a},
$$

and for performance in $S_{2}$ :

$$
\frac{B_{y}}{B_{z}}=\frac{c}{d}\left(\frac{R_{w}}{R_{z}}\right)^{a} .
$$

In Equations 4 and 5, $\mathrm{c}$ is bias and $\mathbf{d}$ is the discriminability of the two $\left(S_{1}, S_{2}\right)$ stimuli. The latter is not the same measure as $d_{r}$, the reinforcer-allocation discriminability in Equation 2. Thus, the Davison-Tustin successivestimulus discriminability will be subscripted $d_{s}$ henceforth. In verbal terms, $d_{r}$ is concerned with the ability of the subject to allocate an obtained reinforcer to responding on one or the other key, whereas $d_{s}$ is concerned with

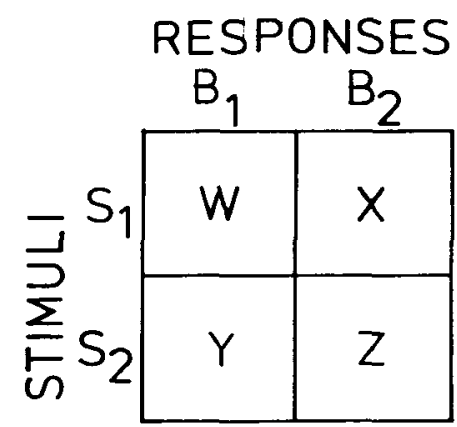

Figure 1. The matrix of events in a yes-no signal-detection procedure. $S_{1}$ and $S_{2}$ are the discriminative stimuli, and $B_{1}$ and $B_{2}$ are the response alternatives. The cells of the matrix are designated $W$, $X, Y$, and $Z$. In the standard procedure, reinforcers are available only in the $W$ and $Z$ cells. 
the ability of the subject to allocate the left-right differentiation correctly to each of the two discriminative stimuli $\left(S_{1}, S_{2}\right)$.

Using Equation 2 to describe the biasing effect of reinforcer frequency in a detection procedure gives two equations which are equivalent to Equations 3 and 4 of the Davison and Tustin (1978) model. We give these in their simplest form, that is, assuming that there are no reinforcers for errors $\left(R_{x}=R_{y}=0\right)$, and omitting the inherent bias (c) parameter, which will simply multiply all left-key reinforcer frequencies. In $S_{1}$ :

$$
\frac{B_{w}}{B_{x}}=\frac{d_{s}\left(d_{r} R_{w}+R_{z}\right)}{d_{r} R_{z}+R_{w}},
$$

and in $S_{2}$ :

$$
\frac{B_{y}}{B_{z}}=\frac{d_{r} R_{w}+R_{z}}{d_{s}\left(d_{r} R_{z}+R_{w}\right)} .
$$

It is usual to arrange that the discriminability of the choice options is maximal, so that $d_{r}$ is large. When this is so, Equations 6 and 7 tend toward:

$$
\frac{B_{w}}{B_{x}}=\frac{d_{s} R_{w}}{R_{z}},
$$

and

$$
\frac{B_{y}}{B_{z}}=\frac{R_{w}}{d_{s} R_{z}} .
$$

Equations 8 and 9 are strict-matching equations $(a=1)$ because we have assumed that $d_{r}=\infty$. From these equations, both bias functions (behavior as a function of reinforcer frequency) and stimulus functions (behavior as a function of stimulus discriminability) can be obtained:

$$
\begin{gathered}
\text { bias }=\frac{B_{w}}{B_{x}} \cdot \frac{B_{y}}{B_{z}}=\left(\frac{R_{w}}{R_{z}}\right)^{2}, \\
\text { discriminability }=\frac{B_{w}}{B_{x}} \cdot \frac{B_{z}}{B_{y}}=d_{s}{ }^{2},
\end{gathered}
$$

as in the Davison-Tustin model when $\mathbf{a}=1$.

For nonasymptotic choice discriminability $\left(d_{r}<\infty\right)$, the bias function is:

$$
\frac{B_{w}}{B_{x}} \cdot \frac{B_{y}}{B_{z}}=\left(\frac{d_{r} R_{w}+R_{z}}{d_{r} R_{z}+R_{w}}\right)^{2}
$$

and the stimulus function is:

$$
\frac{B_{w}}{B_{x}} \cdot \frac{B_{z}}{B_{y}}=d_{s}^{2} .
$$

These equations represent the consistent finding that bias (Equation 10) is independent of discriminability, and that discriminability (Equation 11) is independent of bias in a standard (no error reinforcers) detection procedure (McCarthy \& Davison, 1980). Fitting Equations 6 and 7 to McCarthy and Davison's data, averaged across birds, and using nonlinear regression, gave the following results: For the high-discriminability conditions, $\mathrm{d}_{\mathrm{r}}=3.4$ and $\mathrm{d}_{\mathrm{s}}$ $=22.5$, with $92 \%$ variance accounted for; for the lowdiscriminability conditions, $\mathrm{d}_{\mathrm{r}}=5.2$ and $\mathrm{d}_{\mathrm{s}}=2.3$, with $94 \%$ variance accounted for. The results of fitting Equations 6 and 7 to the individual-subject data are shown in Table 3. The original generalized matching analysis gave: for the high-discriminability conditions, $a=.48$ and $\log \mathrm{d}$ $=1.48(\mathrm{~d}=30.2)$, and for the low-discriminability conditions, $a=.51$ and $\log d=.39(\mathrm{~d}=2.45)$. It is worth noting that the relation between $a$ in Equation 1 and $d_{r}$ in Equation 2 is nonlinear, such that small differences in a can be equivalent to apparently large differences in $d_{r}$. However, the variance across subjects in the estimates of $\mathrm{d}_{\mathrm{r}}$ in the above example (Table 3 ) still supports the conclusion of McCarthy and Davison (1980) that a (hence, $d_{r}$ ) was not significantly different (on a sign test) between the high- and low-discriminability conditions.

This section has shown that if Equation 2 replaces the generalized matching law in the Davison-Tustin (1978) detection theory model for the no-error reinforcement procedure, the theory remains essentially unchanged in its quantitative predictions. The original theory is thus extended to deal with failures of choice discriminability.

\section{Error Reinforcers in Signal Detection}

Can the present theory also deal with the situation in which errors are reinforced (Davison \& McCarthy, 1980; Nevin, Olson, Mandell, \& Yarensky, 1975)? The basic requirement for such a theory is that, taking $S_{1}$ performance only, performance in $S_{1}$ reduces to Equation 6 when only the $\mathrm{W}$ and $\mathrm{Z}$ cells of the matrix of Figure 1

\begin{tabular}{|c|c|c|c|c|}
\hline \multirow[b]{2}{*}{ Bird } & \multicolumn{2}{|c|}{ High Discriminability } & \multicolumn{2}{|c|}{ Low Discriminability } \\
\hline & $d_{s}$ & $d_{r}$ & $d_{s}$ & $d_{r}$ \\
\hline 141 & 37 & 5.8 & 2.1 & 6.7 \\
\hline 142 & 47 & 2.9 & 3.5 & 10.9 \\
\hline 143 & 26 & 2.4 & 2.3 & 8.6 \\
\hline 144 & 20 & 5.2 & 2.8 & 3.9 \\
\hline 145 & 47 & 7.5 & 3.1 & 15.5 \\
\hline 146 & 17 & 3.0 & 1.6 & 5.6 \\
\hline
\end{tabular}
contain reinforcers. It must also reduce to:

$$
\frac{B_{w}}{B_{x}}=\frac{d_{r} R_{y}+R_{x}}{d_{s}\left(d_{s} R_{x}+R_{y}\right)}
$$

when reinforcers are delivered in only the $X$ and $Y$ cells of the matrix. A suitable equation for performance in $S_{1}$ is:

Table 3

Estimates of the Parameters of Equations 6 and 7 for Each of the Subjects Reported by McCarthy and Davison (1980) 


$$
\frac{B_{w}}{B_{x}}=\frac{d_{s}\left(d_{r} R_{w}+R_{z}\right)+d_{r} R_{y}+R_{x}}{d_{r} R_{z}+R_{w}+d_{s}\left(d_{r} R_{x}+R_{y}\right)},
$$

and for performance in $S_{2}$ :

$$
\frac{B_{y}}{B_{z}}=\frac{d_{r} R_{w}+R_{z}+d_{s}\left(d_{r} R_{y}+R_{x}\right)}{d_{s}\left(d_{r} R_{z}+R_{w}\right)+d_{r} R_{x}+R_{y}}
$$

The multiplication of Equations 12 and 13 produces a bias function, and the division of Equation 12 by Equation 13 produces a stimulus function. Both functions are complex, and thus are not written out here, and they indicate that discriminability and bias, as measured in the DavisonTustin model, are not independent when errors are reinforced. Both Davison and McCarthy (1980) and Nevin et al. (1975) showed that discriminability fell when the frequency of error reinforcers was increased. In conformity with these results, Equations 12 and 13 show that discriminability, as conventionally measured, will be zero when equal frequencies of reinforcers are delivered in all four cells of the matrix in Figure 1. The stimulus function from Equations 12 and 13 was fitted to the data, averaged across subjects, reported by Davison and McCarthy. $d_{s}$ was estimated at 6.2 , and $d_{r}$ at 15.4 , with $96 \%$ of the data variance accounted for. This overall $d_{s}$ value is close to the value of 5.9 , which is calculated from the pooled data from their Condition 1, in which errors were not reinforced. The $d_{r}$ value cannot be compared directly with Davison and McCarthy's estimate of $\mathbf{a}$, which was not estimated independently of the model they used. Equations 12 and 13 are thus very good descriptions of detection performance when errors are reinforced.

\section{Multiple-schedule Performance}

In a conventional multiple schedule, defined reinforcers are delivered for the same response $\left(\mathbf{B}_{\mathrm{w}}, \mathbf{B}_{\mathbf{y}}\right.$ in Figure 1) in the presence of two successive discriminative stimuli. Alternate, extraneous, reinforcers are concurrently available during both components $\left(R_{x}=R_{e_{1}}\right.$ and $\left.R_{z}=R_{e_{2}}\right)$, as discussed by Herrnstein (1970) and McLean and White (1983). Thus, Equation 12 becomes:

$$
\frac{B_{w}}{B_{e 1}}=\frac{d_{s} d_{r} R_{w}+d_{s} R_{e 2}+d_{r} R_{y}+R_{e 1}}{d_{r} R_{e 2}+R_{w}+d_{s} d_{r} R_{e 1}+d_{s} R_{y}}
$$

Following McLean and White (1983), we assume that the total output in a component is constant, that is, $B_{w}+B_{e 1}$ $=\mathrm{k}$ (Herrnstein, 1970). Thus:

$$
B_{w}=k\left[\frac{d_{s} d_{r} R_{w}+d_{s} R_{e z}+d_{r} R_{y}+R_{e 1}}{\left(R_{w}+R_{e 1}\right)\left(1+d_{s} d_{r}\right)+\left(R_{y}+R_{e 2}\right)\left(d_{s}+d_{r}\right)}\right] \text {, }
$$

and a similar equation can be written for $\mathrm{B}_{\mathbf{y}}$. From Equa. tion 15 and its equivalent, we can write a complex ex- pression to describe the ratio of component response rates in multiple schedules. Because of its length, it is not reproduced here. If, however, we assume that both stimulus and response-reinforcer discriminability are high, then the present component-response rate ratio equation tends toward:

$$
\frac{B_{w}}{B_{y}}=\left(\frac{R_{w}}{R_{y}}\right)\left(\frac{R_{y}+R_{e 2}}{R_{w}+R_{e 1}}\right)
$$

Equation 16 is the nonpower function equivalent of McLean and White's (1983) quantitative description of performance in multiple schedules, which is:

$$
\frac{B_{w}}{B_{y}}=\left(\frac{R_{w}{ }^{a}}{R_{y}{ }^{a}}\right)\left(\frac{R_{y}{ }^{a}+R_{e 2}{ }^{a}}{R_{w}{ }^{a}+R_{e 1}{ }^{a}}\right) .
$$

Given that $d_{r}$ in the present model replaces the power function in the generalized matching law, and given that McLean and White showed that Equation 17 was a good fit to multiple VI VI schedule data, Equation 16 is very likely to be successful. McLean and White reported data from multiple-concurrent schedules, which allowed all the reinforcer rates required by Equation 16 to be measured as rates of food reinforcers. McLean and White's Experiment 1 data, averaged across birds, were fit using Equation 15 and its equivalent for $B_{y}$ to predict the ratio $B_{w} / B_{y}$. The obtained parameter estimates were: $d_{s}=34$ and $d_{r}$ $=17$, with $99 \%$ of the variance accounted for. The value of $a$ in Equation 17 was estimated at .85 (averaged over the four birds) by McLean and White, which represents a $d_{r}$ value of between 15 and 38 (Table 2). A direct nonlinear fit of Equation 17 (the McLean and White model) gave an estimate of $\mathbf{a}=.81\left(\mathrm{a} \mathrm{d}_{\mathrm{r}}\right.$ value of a little above 15 ), with $98 \%$ of the variance accounted for. It is worth noting, at this point, that Equation 16 (the McLean-White model without an exponent) accounted for $79 \%$ of the data variance.

It is much more difficult to fit the present model directly to a conventional multiple-schedule data because $R_{e_{1}}$ and $R_{e 2}$ are variables, rather than constants, and their values are not experimentally measured. For the purposes of the present analysis, however, we may, like Herrnstein (1970), make the simplifying assumption that $R_{\mathrm{e}_{1}}=R_{\mathrm{e}_{2}}$ $=R_{e}$, a constant. Under these conditions, the equation for component response-rate ratios may be fitted iteratively. Using the data provided by Charman and Davison (1982), averaged across birds, we estimate $d_{s}$ as $7.3, d_{r}$ as 1.3 , and $R_{e}$ as 1.2 reinforcers per minute, with $94 \%$ of the variance accounted for. This fit, though, is rather unsatisfactory due to the small number of data points (5) in relation to the substantial number of free parameters and the fact that the assumption that $R_{e_{1}}=R_{e_{2}}=R_{e}$ strongly affects the estimation of the "undermatching" $\left(d_{r}\right)$ parameter. In light of the report by Charman and Davison (1983), which showed very high stimulus dis- 
criminability in multiple schedules, we believe that the $\mathrm{d}_{s}$ parameter is also underestimated in the above fit. Because of the problems in measuring $R_{\mathrm{e}_{1}}$ and $R_{\mathrm{e}_{2}}$ in standard multiple schedules, it is clearly preferable to study multiple-concurrent schedule performance (as did McLean $\&$ White, 1983) in order to measure and manipulate extraneous reinforcer rates.

It may also be possible to modify further the present model in order to account for the finding that behavior in a multiple-schedule component is more affected by the conditions in the component that follows that component than by the conditions in the component that precedes that component (the following-component effect; Williams, 1981). Such an effect may result from differences in proactive and retroactive effects of components on stimulus or response-reinforcer contingency discriminability.

\section{Responding on Single VI Schedules}

Equation 15, in conjunction with the assumption made by Herrnstein (1970) that the total response rate is constant (k), can be used to describe response rates in single schedules. We will assume that reinforcer rates in successive parts of a schedule are indiscriminable, but with a caveat. It is possible that, for instance, the occurrence of the smallest interreinforcer interval is discriminable to a subject, and that it signals a particular next interreinforcer interval. Thus, $d_{s}$ may not be 1 (no discriminability) and $R_{y}$ may not equal $R_{w}$ in certain arrangements of schedules. $A d_{s}$ of 1 may thus be appropriate only for a VI schedule in which the sequence of intervals is randomized from an exponential distribution (see Taylor \& Davison, 1983, for the suggestion that arithmetic VI schedule performance is different from exponential VI schedule performance). Assuming $d_{s}$ is one, $R_{e_{1}}=R_{e_{2}}$ $=\mathbf{R}_{\mathrm{e}}, \mathbf{R}_{\mathbf{w}}=\mathbf{R}_{\mathbf{y}}, \mathbf{R}_{\mathrm{x}}=\mathbf{R}_{\mathrm{z}}$, and that $B_{\mathrm{w}}+\mathrm{B}_{\mathrm{e} \mathbf{1}}=\mathrm{k}$ (Herrnstein, 1970), Equation 15 becomes:

$$
B_{w}=k\left[\frac{d_{r} R_{w}+R_{e}}{\left(R_{w}+R_{e}\right)\left(1+d_{r}\right)}\right] .
$$

Equation 18 tends toward Herrnstein's equation for singleschedule VI performance when $d_{r}$ is large:

$$
B_{w}=k\left[\frac{R_{w}}{R_{w}+R_{e}}\right] .
$$

To the extent that the subject does confuse the defined response-reinforcer contingencies with the extraneous response-reinforcer contingencies, the data will deviate from Herrnstein's hyperbola.

Clearly, some schedule types arrange discriminable periods of different reinforcer rates (e.g., fixed-ratio and fixed-interval schedules), and performance on these schedules may be describable by an equation that is the singleschedule equivalent to Equation 12.

\section{Conclusions}

We offer Equation 2 as a description of concurrent- schedule performance which is preferable to the generalized matching law (Equation 1), and we have shown how the acceptance of Equation 2 leads to a coherent and consistent set of equations which are good descriptors of performance in the various procedures in which the matching and generalized matching relations have been used. The reinterpretation of sensitivity to reinforcement (a) as contingency discriminability leads to a wide range of predictions about conditions that will affect "sensitivity," which may be experimentally investigated.

Davison and Tustin (1978) and McCarthy and Davison (1981) conceived of their work as deriving detection theory from generalized matching, and thus providing a theory of bias (generalized matching) for detection theory and a theory of stimulus effects (discriminability) for behavior theory. The present paper has taken this approach a logical step forward and dealt with reinforcement itself as a detection process. The overall theory now deals in a coherent manner with the detection of environmental events and their effects on behavior.

\section{REFERENCES}

Allen, C. M. (1981). On the exponent in the "generalized"' matching equation. Journal of the Experimental Analysis of Behavior, 35, 125-127.

BaUM, W. M. (1974). On two types of deviation from the matching law: Bias and undermatching. Journal of the Experimental Analysis of Behavior, 22, 231-242.

BAUM, W. M. (1979). Matching, undermatching, and overmatching in studies of choice. Journal of the Experimental Analysis of Behavior, 39, 269-281.

BAUM, W. M. (1982). Choice, changeover, and travel. Joumal of the Experimental Analysis of Behavior, 38, 35-49.

Charman, L., \& Davison, M. (1982). On the effects of component duration and component reinforcement rate in multiple schedules. Journal of the Experimental Analysis of Behavior, 37, 417-439.

Charman, L., \& Davison, M. (1983). Undermatching and stimulus discriminability in multiple schedules. Behaviour Analysis Letters, 3 , 77-84

Davison, M. C., \& Hunter, I. W. (1976). Performance on variableinterval schedules arranged singly and concurrently. Journal of the Experimental Analysis of Behavior, 25, 335-345.

DavisOn, M., \& MCCARTHY, D. (1980). Reinforcement for errors in a signal-detection procedure. Journal of the Experimental Analysis of Behavior, 34, 35-47.

Davison, M. C., \& Tustin, R. D. (1978). The relation between the generalized matching law and signal-detection theory. Journal of the Experimental Analysis of Behavior, 29, 331-336.

DE Villiers, P. A. (1977). Choice in concurrent schedules and a quantitative formulation of the law of effect. In W. K. Honig \& J. E. R Staddon (Eds.), Handbook of operant behavior. Englewood Cliffs, N.J: Prentice-Hall.

DE VILliERs, P. A. (1980). Toward a quantitative theory of punishment Journal of the Experimental Analysis of Behavior, 33, 15-25.

FARLEY, J. (1980). Reinforcement and punishment effects in concurrent schedules: A test of two models. Joumal of the Experimental Analysis of Behavior, 33, 311-326.

Findley, J. D. (1958). Preference and switching under concurrent scheduling. Journal of the Experimental Analysis of Behavior, 1, 123-144.

Green, D. M., \& Swets, J. A. (1974). Signal-detection theory and Psychophysics. N.Y: Wiley.

Herrnstein, R. J. (1970). On the law of effect. Journal of the Experimental Analysis of Behavior, 13, 243-266.

KILleEN, P. R. (1978). Superstition: A matter of bias, not detectability. Science, 199, 88-90. 
KILleEN, P. R. (1981). Learning as causal inference. In M. L. Commons \& J. A. Nevin (Eds.), Quantitative analysis of behavior, Vol. 1: Discriminative properties of reinforcement schedules. Cambridge, MA: Ballinger.

LATTAL, K. A. (1975). Reinforcement contingencies as discriminative stimuli. Journal of the Experimental Analysis of Behavior, 23, 241-246.

LoBB, B., \& Davison, M. C. (1975). Performance in concurrent interval schedules: A systematic replication. Journal of the Experimental Analysis of Behavior, 24, 191-197.

MCCARThY, D., \& Davison, M. (1980). Independence of sensitivity to relative reinforcement rate and discriminability in signal detection. Journal of the Experimental Analysis of Behavior, 34, 273-284.

McCarthy, D., \& Davison, M. (1981). Toward a behavioral theory of bias in signal detection. Perception \& Psychophysics, 29, 371-382.

McLean, A. P., \& WhrTe, K. G. (1983). Temporal constraint on choice: Sensitivity and bias in multiple schedules. Journal of the Experimental Analysis of Behavior, 39, 405-426.

Miller, J. T., Saunders, S. S., \& Bourland, G. (1980). The role of stimulus disparity in concurrently available reinforcement schedules. Animal Learning \& Behavior, 8, 635-641.

Nevin, J. A. (1967). Effects of reinforcement scheduling on simultaneous discrimination performance. Journal of the Experimental Analysis of Behavior, 10, 251-260.

Nevin, J. A., Olson, K., Mandell, C., \& Yarensky, P. (1975). Differential reinforcement and signal detection. Journal of the Experimental Analysis of Behavior, 24, 355-367.

Pliskoff, S. S., \& Fetterman, J. G. (1981). Undermatching and overmatching: The fixed-ratio changeover requirement. Journal of the Experimental Analysis of Behavior, 36, 21-27.
PreLeC, D. (1984). The assumptions underlying the generalized matching law. Journal of the Experimental Analysis of Behavior, 41, 101-107.

Rachlin, H. C., Kagel, J. H., \& Battalio, R. C. (1980). Substitutability in time allocation. Psychological Review, 87, 355-374.

TAYLOR, R., \& DAvisON, M. (1983). Sensitivity to reinforcement in concurrent arithmetic and exponential schedules. Journal of the Experimental Analysis of Behavior, 39, 191-198.

ToDorov, J. C. (1971). Concurrent performances: Effects of punishment contingent on the switching response. Journal of the Experimental analysis of Behavior, 16, 51-62.

WEARDEN, J. H. (1980). Undermatching on concurrent variable-interval schedules and the power law. Journal of the Experimental Analysis of Behavior, 33, 149-152.

WEARDEN, J. H. (1983). Undermatching and overmatching as deviations from the matching law. Journal of the Experimental Analysis of Behavior, 40, 332-340.

WEARDEN, J. H., \& Burgess, I. S. (1982). Matching since Baum (1979). Journal of the Experimental Analysis of Behavior, 38, 339-348.

White, K. G., \& REDMAN, S. (1983). Free-operant forgetting: Delayed stimulus control of multiple-schedule performance. Journal of the Experimental Analysis of Behavior, 39, 129-133.

Williams, B. A. (1975). The blocking of reinforcement control. Journal of the Experimental Analysis of Behavior, 24, 215-225.

Williams, B. A. (1981). The following schedule of reinforcement as a fundamental determinant of steady-state contrast in multiple schedules. Journal of the Experimental Analysis of Behavior, 35, 293-310.

(Manuscript received May 10, 1984;

revision accepted for publication September 18, 1984.) 